\title{
Analysis of Selected Quality-of-Life Indicators in Patients Undergoing Comprehensive Neurorehabilitation After Ischemic Stroke- A Single-Centre Cross-Sectional Study
}

\author{
Łukasz Argier ${ }^{1}$ \\ ${ }^{1}$ Saint Hedwig Provincial Specialist Neuropsychiatry Hospital in Opole \\ Correspondence to: Łukasz Argier, \\ Saint Hedwig Provincial Specialist Neuropsychiatry Hospital in Opole, Wodociagowa 4, 45-221 Opole \\ argier@o2.pl
}

DOI: https://doi.org/10.5114/phr.2021.104602

Received: 30.01.2021 Reviewed: 07.02.2021 Accepted: 07.02.2021

\section{Abstract}

Aims: To assess the quality of life (QOL) of people after ischemic stroke and to determine the influence of gender and side of hemiparesis on the patient's self-perception of QOL.

Materials and methods: The study involved 50 patients who were divided into 2 groups based on gender. The modified version of the Stroke-Specific Quality of Life Scale (SSQOL) was used to assess QOL, and the Modified Rivermead Motor Assessment (MRMA), the Modified Barthel Index (MBI) and the Upper Extremity Modified Motor Assessment (UEMMA) were used to assess the effects of rehabilitation. The comprehensive neurorehabilitation programme was conducted in an outpatient clinic over a period of 3 weeks, 5 days a week, and included specialised physiotherapy, speech therapy, psychotherapy, and occupational therapy. Exercises according to the proprioceptive neuromuscular facilitation (PNF) and Bobath methods were performed.

Results: After the applied comprehensive neurorehabilitation, an improvement was obtained in all examined indicators $(\mathrm{p}<0.05)$.

Conclusions: The side of hemiparesis was found to be related to self-perceived QOL. The patient's gender had no effect on the assessed indicators. Similarly, there was no correlation between the patients' age and QOL parameters.
Key words

neurorehabilitation, physiotherapy, stroke, quality of life 


\section{Introduction}

Quality of life (QOL) is one of the key determinants of the effectiveness of comprehensive rehabilitation. This type of rehabilitation becomes effective only when the patient returns home after the treatment and is able to function independently. Therefore, the main goal of the process is to restore the lost functions as much as possible. Neurorehabilitation for stroke patients is particularly complex [1].

The difficulty in defining the results of physiotherapeutic interventions in neurorehabilitation unambiguously is due to the fact that each patient has a period of natural return of function and self-compensation. Therefore, QOL is a very measurable tool, as it includes functional ability, emotional state, life satisfaction, and self-assessment of health [2].

\section{Aims}

The aim of this study is to assess the QOL of people after ischemic stroke and determine the influence of gender and side of hemiparesis on the patient's self-perception of QOL.

\section{Materials and Methods}

The study was conducted among patients who had experienced ischemic stroke and were treated at the Department of Daily Rehabilitation of the St Jadwiga's Regional Specialist Neuropsychiatric Centre in Opole, Poland.

The inclusion criteria for the study were: (1) ischemic stroke, (2) first or subsequent stroke, (3) duration of 1-6 months after stroke, (4) mental and verbal communication skills allowing participation in the study, (5) subject's age above 18 years, (6) informed consent to participate in the study, and (7) physical ability that allowed for a 6-hour stay in a sitting position during physiotherapy, occupational therapy, speech therapy and psychotherapy.

The exclusion criteria for the study were: (1) haemorrhagic stroke, (2) duration more than 6 months since stroke, (3) mental and verbal communication disturbances not allowing participation in the study, (4) age below 18 years, (5) lack of patient consent to participate in the study, (6) physical ability that did not allow for a 6-hour stay in a sitting position and (7) other neurological and internal conditions that could affect the validity of the conclusions.

The study involved 50 patients, who were divided by gender into two groups. Group I consisted of 24 women aged 31-86 years (mean age 60 years). A proportion of $54 \%$ of the subjects lived in a city, $33 \%$ had pursued higher education, 54\% were retired and $41 \%$ were married. The majority of women, $62 \%$, had right-sided hemiparesis, while $58 \%$ did not receive regular physiotherapy. Group II consisted of 26 men in the age range of 38-81 years (mean age 62). A proportion of $69 \%$ lived in a city, $31 \%$ had vocational education, $50 \%$ were retired and the same percentage were married. Most of the men had right-sided hemiparesis (65\%), and 50\% did not receive regular physiotherapy.

A modified version of the Stroke Specific Quality of Life Scale (SSQOL) was used to assess the QOL of patients after ischaemic stroke. The tool consists of 47 questions divided into 12 subscales: self-care, vision, language, mobility, work, upper limb function, thinking, personality, mood, family roles, social roles, energy. For each question, there was one of five answers to choose, with one answer scored 1 indicating an inability to perform certain activities and answer scored 5 indicating no difficulties. The modified version of SSQOOL scoring was as follows, in questions 1 to 47 there were 5 possible answer options, where 1 (total inability) $=0$ points; 2 (great difficulty) $=1$ point; 3 (medium difficulty) $=2$ points; 4 (mild difficulty $)=$ 3 points; and 5 (no difficulty) $=4$ points .

The following three research tools were used to assess the effects of rehabilitation:

1. Modified Rivermead Motor Assessment (MRMA), where 0-40 points can be scored in the corresponding categories: group I: 0-10 points - very severe condition, totally dependent; group II: 11-20 points - severe condition, 
requires assistance; group III: 21-30 points medium condition; and group IV: $3-40$ points - mild condition.

2. Modified Barthel Index (MBI), where 0-20 points can be scored in the corresponding categories: group I: 0-6 points - severe condition; group II: 7-14 points - medium condition; and group III: $15-20$ points - mild condition.

3. Upper Extremity Modified Motor Assessment (UEMMA), where 0-32 points can be scored and it includes 16 questions addressing the upper limb function and movement.

The comprehensive neurorehabilitation programme was conducted on an outpatient clinic over a period of 3 weeks, 5 days a week and included specialised physiotherapy, speech therapy, psychotherapy, and occupational therapy. Exercises according to the PNF and Bobath methods were performed.

Results were analysed using STATISTICA software (Dell Inc., Tulsa, USA). Student's t-test, U-Mann
Whitney test were applied. An analysis of the study groups was performed regarding the effects of gender and hemiparesis side on self-perceived QOL. Differences were considered statistically significant at $\mathrm{p}<0.05$.

\section{Results}

The results of all indicators showed a significant improvement compared to the pre-treatment condition but no statistically significant differences with gender. Selected differences are shown below. A statistically significant difference in the answers to the question on eating problems was shown $(\mathrm{p}<0.05)$. This indicates that the people with right-sided hemiparesis had greater difficulties with eating than those with left-sided hemiparesis. This may be due to the fact that the right is the dominant side of the body or that hemiparesis was also present in the facial muscles, which made eating more difficult (Figure 1).

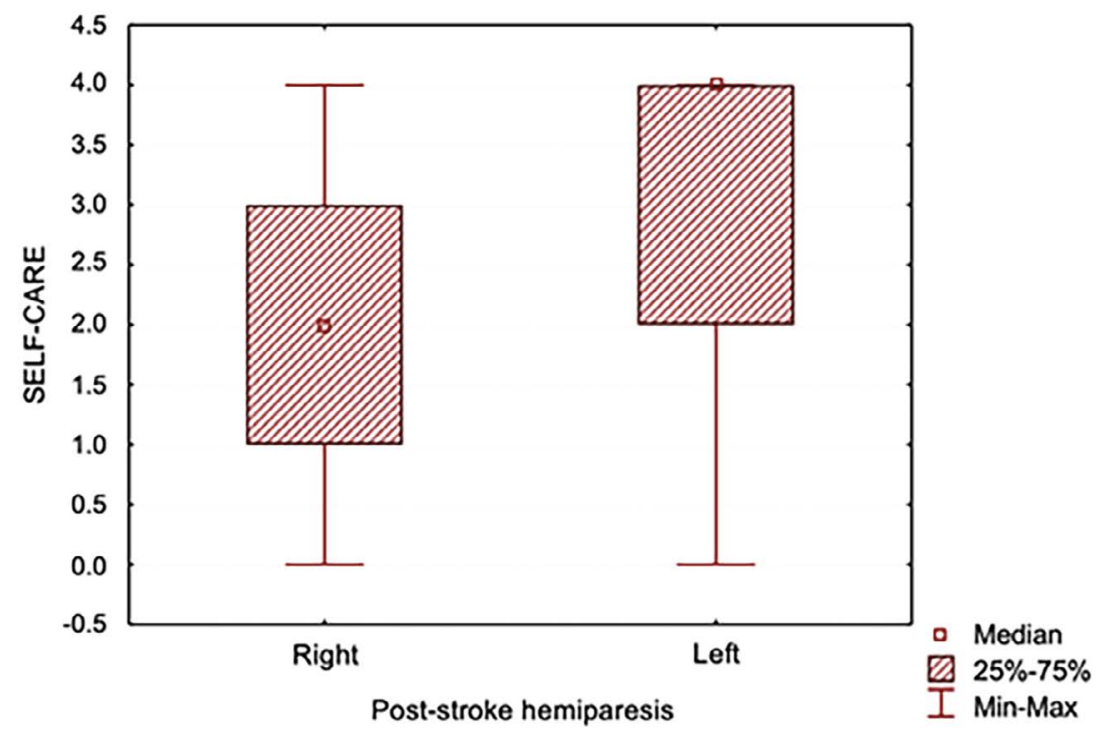

Figure 1. Variation in scores across responses to the question: Have you had difficulties with self-care during meals?

A statistically significant difference was also observed for the answers to the question on difficulties in reaching for things due to impaired vision $(\mathrm{p}<0.05)$. This may be due to the presence of hemianopsia in people with right-sided hemiparesis or to the more advanced age of these subjects, which may have also negatively affected their quality of vision (Figure 2). 


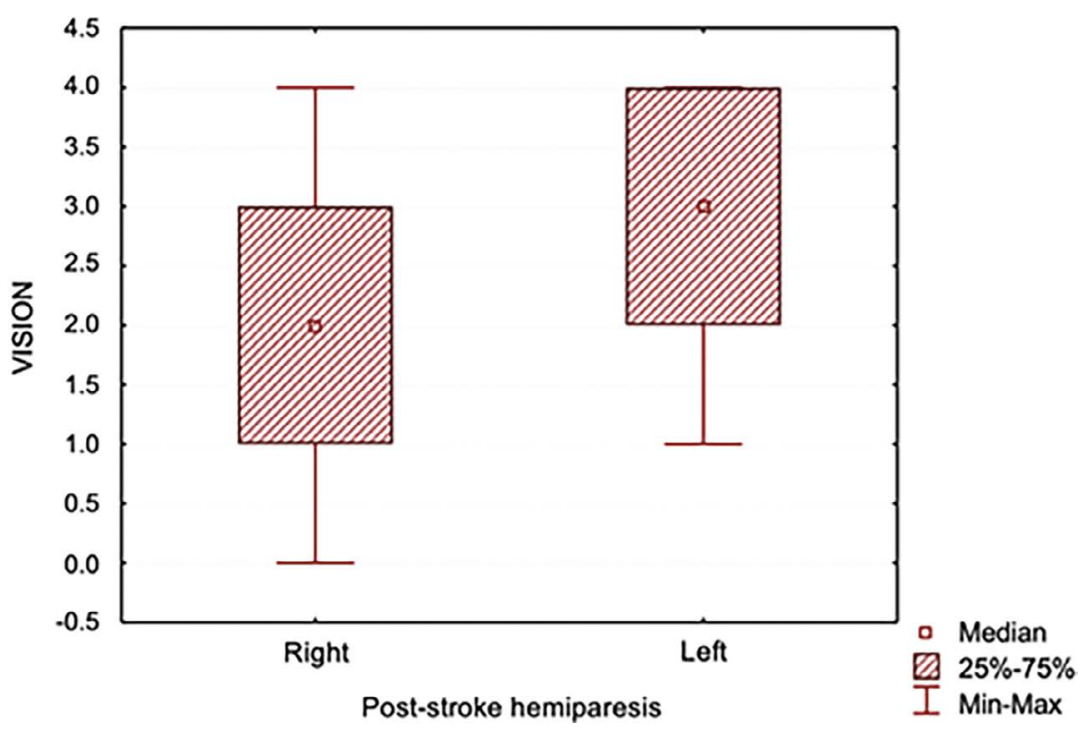

Figure 2. Variation in scores across responses to the question: Have you had difficulties while reaching for things because of impaired vision?

It was found that subjects with right-sided hemiparesis had significantly more difficulties with speech, which was unclear and words were distorted $(\mathrm{p}<0.05)$. This may indicate that in most people, the stroke affected the dominant left cerebral hemisphere where the speech areas are located. This pattern was also observed in the case of speech perception (Figure 3).

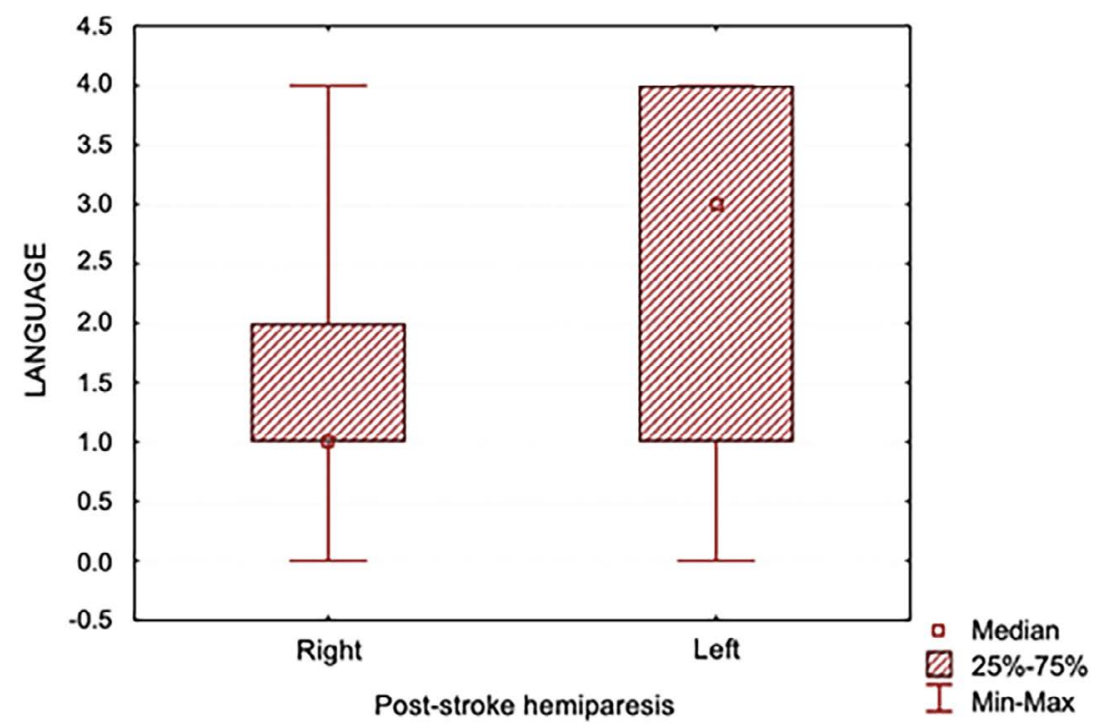

Figure 3. Variation in scores across responses to the question: Did you have difficulties with speech, was language unclear and were words distorted? 
It was documented that subjects with right-sided hemiparesis had more difficulties in writing than those with left-sided hemiparesis $(\mathrm{p}<0.05)$. This may suggest that most strokes were caused by occlusion of the middle cerebral artery, which supplies the area responsible for upper limb functions (Figure 4).

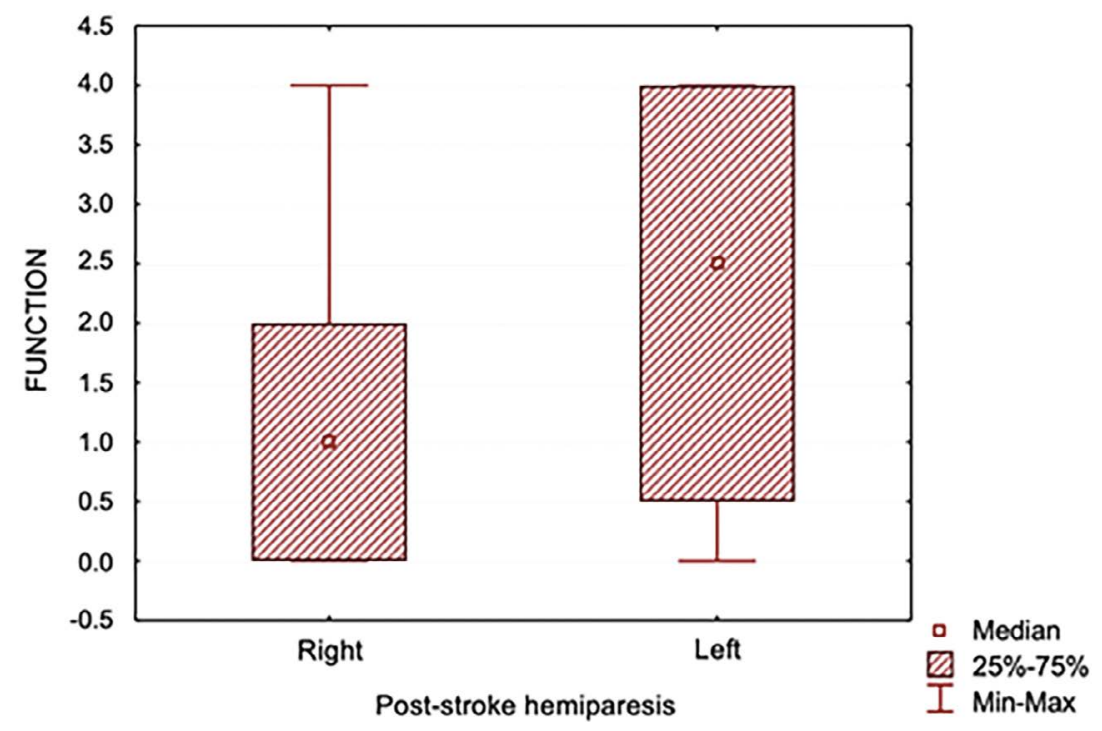

Figure 4. Variation in scores across responses to the question: Have you had difficulties with upper limb function during handwriting or typesetting?

There was a statistically significant difference in the answer to the question concerning energy levels, which were lower in people with left-sided hemiparesis $(\mathrm{p}<0.05)$. This may be due to the fact that these people had a better general fitness le- vel and were therefore able to perform heavier daily activities. This could have led to higher fatigue, although the reason may also be the occurrence of post-stroke depression (Figure 5).

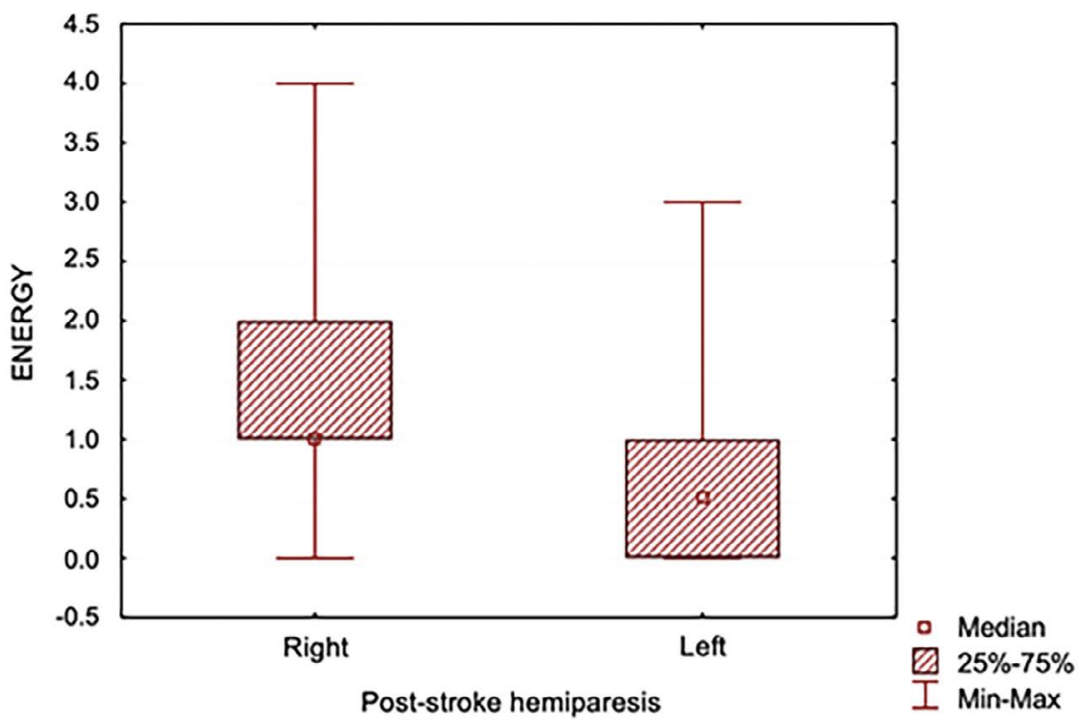

Figure 5. Variation in scores across responses to the question: Have you had difficulties related to fatigue most of the time? 


\section{Discussion}

The assessment of QOL in post-stroke patients is a challenging issue. It includes many areas of life which are subjectively interpreted by the patient. The statistical analysis carried out in the present study indicates lower scores for the assessed indicators among subjects with right-sided hemiparesis. This may be due to the left-sided localisation of the damage to the cerebral hemisphere, which is associated with depressive symptoms, impairments in speech, writing, analytical thinking, control of complex motor activities, learning, and memory $[3,4]$.

A study by Baumann et al. [5] showed that people with right-sided hemiparesis have more difficulties with activities such as eating and writing than people with right-sided hemiparesis. In turn, Giaquinto et al. [6] showed that women suffer more from stroke than men due to a psychological mechanism called 'narcissistic injury'. The same researchers also pointed out that women with even low levels of disability have problems with social interaction, making their QOL significantly worse than that of the male population.

Different conclusions were drawn by Berges et al. [7], who found that women with similar pain intensity as men were more satisfied with their QOL. According to the authors, this may be related to the fact that women are more active in seeking help to relieve pain and are more likely to accept pharmacological treatment. This is confirmed by Portuguese researchers who, after reviewing the literature from 2008 to the present, concluded that women have significantly more knowledge about stroke than men, which may affect their better perception of QOL [5].

In the present study, no relationships between QOL and gender were found. This may be related to the fact that the number of subjects was too low, so the results were not statistically significant. However, as can be seen from the above reports, there is no consensus on this issue in the literature.

Family support and involvement in the rehabilitation process is a very important factor in post-stroke recovery. Clinical practice proves that stroke survivors who have the support of their relatives, especially their partners, experience faster recovery and a significant improvement in their QOL. Haley et al. [8] noted that patients who live alone are more likely to suffer from post-stroke depression than patients who live with family. This is due to the fact that social isolation is one of the main risk factors for post-stroke depression.

Mayo et al. [9] demonstrated that people who showed a greater intention for social participation have a better QOL. An association of depression with damage to the left-brain hemisphere has been noted, but the authors acknowledge that in practice, the link between the influence of the side of hemiparesis on the occurrence of post-stroke depression is not so noticeable. In addition, it has been noted that greater severity of depressive symptoms is accompanied by more extensive brain damage.

Our paper showed that people with left-sided hemiparesis are more likely to be fatigued during the day than those with right-sided hemiparesis, which may indicate the presence of post-stroke depression. This fatigue may also be associated with the increased functional capacity of patients with left-sided hemiparesis: taking up a greater number of activities may induce fatigue.

A study conducted by Kwok et al. [10] found that post-stroke survivors have significantly more problems in their relationships with family and friends than healthy individuals. It was also observed that sexual dysfunction, which is common after stroke, has a significant impact on the worsening of partner relationships.

This study shows that people with right-sided hemiparesis have substantial difficulties with speech, which is unclear and distorted. Patients often declare that they have difficulties in having their speech understood by other people, which negatively affects interpersonal contacts and reduces QOL. The effect of young age on the perception of QOL has not been proven in this study, which may be due to the presence of too few young people in the study population-in which the mean age was 60 years. 


\section{Conclusions}

This study revealed that the side of hemiparesis is related to self-perceived QOL. The patient's gender had no effect on the assessed indicators.
Similarly, no correlation was found between the patients' age and QOL parameters.

\section{References}

1. Liu Z, Zhou X, Zhang W, Zhou L. Factors associated with quality of life early after ischemic stroke: the role of resilience. Top Stroke Rehabil. 2019; 26 (5): 335-341.

2. Cumming TB, Brodtmann A, Darby D, Bernhardt J. The importance of cognition to quality of life after stroke. J Psychosom Res. 2014; 77 (5): 374-379.

3. Loubinoux I, Kronenberg G, Endres M, Schumann-Bard P, Freret T, Filipkowski RK et al, Post-stroke depression: mechanisms, translation and therapy. J Cell Mol Med. 2012; 16 (9): 1961-9.

4. Rodgers H. Stroke. Handb Clin Neurol. 2013; 110: 427-433.

5. Baumann M, Lurbe K, Leandro ME, Chau N. Life satisfaction of two-year post-stroke survivors: effects of socio-economic factors, motor impairment, Newcastle stroke-specific quality of life measure and World Health Organization quality of life: brief of informal caregivers in Luxembourg and a rural area in Portugal. Cerebrovasc Dis. 2012; 33 (3): 219-230.
6. Giaquinto S, Giachetti I, Spiridigliozzi C, Nolfe G. Quality of life after stroke in a rehabilitation setting. Clin Exp Hypertens. 2010; 32 (7): 426-430.

7. Bergés IM, Ottenbacher KJ, Kuo YF, Smith PM, Smith D, Ostir GV. Satisfaction with quality of life poststroke: effect of sex differences in pain response. Arch Phys Med Rehabil. 2007; 88 (4): 413-417.

8. Haley WE, Roth DL, Kissela B, Perkins M, Howard G. Quality of life after stroke: a prospective longitudinal study. Qual Life Res. 2011; 20 (6): 799-806.

9. Mayo NE, Wood-Dauphinee S, Côté R, Durcan L, Carlton J. Activity, participation, and quality of life 6 months poststroke. Arch Phys Med Rehabil. 2002; 83 (8): 1035-1042.

10. Kwok T, Lo RS, Wong E, Wai-Kwong T, Mok V, Kai-Sing W. Quality of life of stroke survivors: a 1-year follow-up study. Arch Phys Med Rehabil. 2006; 87 (9): 1177-1182. 